\title{
Review of Temperature Measurement Techniques
}

\author{
Husain Al Hashimi ${ }^{1}$, Omar Chaalal ${ }^{2 *}$, Marouane Chaalal ${ }^{3}$ and Hameed Muhamed ${ }^{4}$ \\ ${ }^{1}$ Consultant Mechanical Engineering, UAE \\ ${ }^{2}$ Abu Dhabi University College of Engineering, Chemical Engineering, UAE \\ ${ }^{3}$ Engineer Fluor Gulf Plant, UAE \\ ${ }^{4}$ Sheridan College, Canada \\ *Corresponding author: Omar Chaalal, Abu Dhabi University College of Engineering, Chemical Engineering, UAE
}

\section{ARTICLE INFO}

Received: 蔧 December 01, 2021

Published: 幽 December 13, 2021

Citation: Husain Al Hashimi, Omar Chaalal, Marouane Chaalal, Hameed Muhamed. Review of Temperature Measurement Techniques. Biomed J Sci \& Tech Res 40(3)-2021. BJSTR. MS.ID.006465.

\section{ABSTRACT}

Rapid development in the area of temperature measurement techniques arises from various applications, such as the sessile drop, which is of interest in several fields including coating, combustion, and cooling facilities. Accurate depiction of the physical phenomenon entails improvement in the temperature measurement resolution, where average temperature measurements fail to capture, due to their spatial limitation. In the current paper, various optical techniques are reviewed in contrast to conventional measurement approaches, such as thermocouples and microheater arrays. The optical techniques include Infrared thermography and fluorescing materials, such as quantum dots and Temperature sensitive paint. The advantages and shortcomings of such techniques are examined in depth, with respect to their applications in previous works.

\section{Introduction}

Phase change phenomenon involves instantaneous variations of the local heat transfer, which is coupled to the unsteady fluid currents overlying the surface. An example is the sessile drop, which is of interest in several fields including coating, combustion, and cooling facilities. Understanding of these mechanisms requires fine spatial and temporal measurements, which is essential for applications associated with design optimization and safety consideration of a process. Such consideration is crucial within the operations of boilers, for instance, where the evolved heat flux is restricted by the boiling crisis. This operational regime is often associated with equipment failure. Within the miniaturized electronics applications such as transistors, high heat fluxes up to $200 \mathrm{~W} / \mathrm{cm}^{2}$ can be liberated from such instruments, where a low wall superheat is desired with respect to the cooling fluid. Thus, operating within the correct boiling regime becomes paramount in the thermal management of the operating equipment [1-4]. In addition, some of the proposed models are not well validated due to the limited resolution of the available data, which might bring about a misinterpretation of the phase change phenomenon under study. Such situations can be encountered with respect to models based on point measurements, which cannot resolve fine spatial resolutions associated with phase change phenomenon [5-6]. In the current paper, some of the relevant temperature measurement techniques are provided, which include point measurement techniques and IR thermography. A review of fluorescing materials and their usage within temperature measurement applications are given by the end of the paper.

\section{Point Measurements}

A conventional point measurement technique is the thermocouple, which operates on the basis of Seebeck effect. In principle, two dissimilar metals are connected at a junction, which generates a small voltage with respect to a given temperature. Such technique was used to acquire average heat flux measurement over a surface of interest [6]. In parallel to this technique, Truong [7] considered using a heat fluxmeter, so as to evaluate the heat 
transfer coefficient associated with a heat sink, via Newton's law of cooling. The drawback of such methods is that its usage is limited to quasi-steady state regime, which cannot be used for complex flow phenomena, due to multidimensionality and the instantaneous local changes of the fluid-wall interaction [6-7]. In case of the heat fluxmeter, significant measurement error can incur, in case the thermal properties of the instrument was not well quantified [7].

\section{Resistive Based Sensors}

In the past decades, thermal resistance-based sensors has been the focus of wide of variety of applications. These include thermal actuator, flow rate and temperature measuring devices, and sensors pertaining to gas monitoring within food logistics [8-10]. Several studies focused on developing high compact microheater sensors, with competitive spatial resolution performance. Such advances were permitted with development of microfabrication, which enable one to realize fine features as small as a submicron length. Such sensors can play a crucial role in detailed examination of adverse flow condition, as in phase change application for instance, which can be achieved by its instantaneous measurement at multipoint resolution and its conformity to nonplanar surfaces [11-14]. Among the various works on resistive sensors, is the one done by Guereca [15] in the field of microelectromechanical systems, which was used in boiling application. The instrument used in Guereca [15] study was fabricated via ion beam milling and photolithography, which had a dual function of providing heat to surrounding fluid and for temperature measurement purposes. The latter used to measure the nucleation temperature, based on sudden changes in the observed temperature, with respect to the temperature coefficient of the instrument resistance [15]. Figure 1 depicts the microheater array used by Demiray and Kim [16] within FC-72 pool boiling experiment.
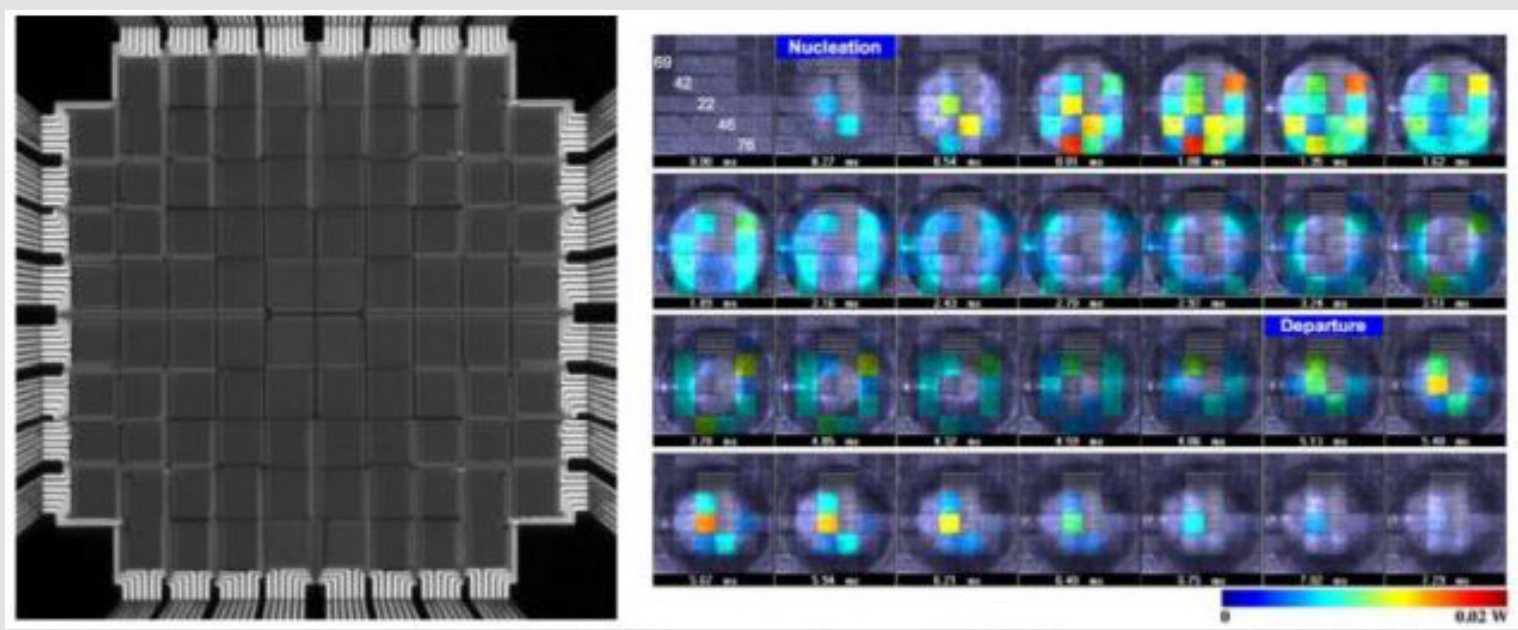

Figure 1: Micro heater array for pool boiling experiment (left), heat transfer distribution under nucleating bubble at micro heater surface (right) [16].

Some of the major challenges with respect to the sensor's fabrication regard the dimensional scale difference between the patterned lead connection and the deposited sensor metal which is one of the main causes for electrical disconnection, due to the sensor's breakage along the lead's edges. Such issues are further complicated if an adhesion layer exist between the flexible substrate and the pattern leads. In addition, it is crucial to include a thermal insulation within the metal sensor design, which can affect its performance criteria, such as measurement sensitivity and resolution [11-12,17-19]. Also, some studies have indicated deviation of the material properties from bulk metal value for thicknesses close to the mean free path. For instance, Siegel, et al. [20] observed an inverse relation between resistance and temperature for gold thickness of several $\mathrm{nm}$, as opposed to the proportional correlation between resistance and temperature for bulkier gold thicknesses. Inhomogeneity in the deposited sensor can lead to large temperature gradient on the sensor's surface, which can affect the integrity of the temperature measurement. For instance, Guereca [15] noticed that the acquired temperature measurement was lower for thinner fabricated resistive sensor. Lastly and not least, such temperature measurement requires direct contact with fluid of interest, and it has an element of Joule's heating. Thus, there is some form of intrusion upon the investigated phenomenon, which might induce some form of error.

\section{Non-Contact Local Temperature Measurement Techniques}

A breakthrough in surface temperature measurement has been achieved recently, via infrared (IR) technology, which permitted local heat flux measurements at fine spatial resolution. Such 
technique proved to be of an essence in proper understanding of heat transfer mechanism, and to further elaborate existing models and correlations.

\section{Infrared Thermography}

Several studies utilized Infrared (IR) technique in order to resolve the local wall heat transfer in both pool and flow boiling applications. An example is the study conducted by Scammell and Kim [21], where they examined the effect of vortex shedding upon the local wall heat transfer in flow boiling applications. A schematic of the flow boiling test section is shown in Figure 2. The IR measurements in such studies are based on black coating of the wall surface, adjacent to the working fluid, which acted as temperature markers for the IR camera. The temperature profile across the wall was deduced numerically, in a coupled radiationconduction problem from which the local heat flux profile was resolved [21].

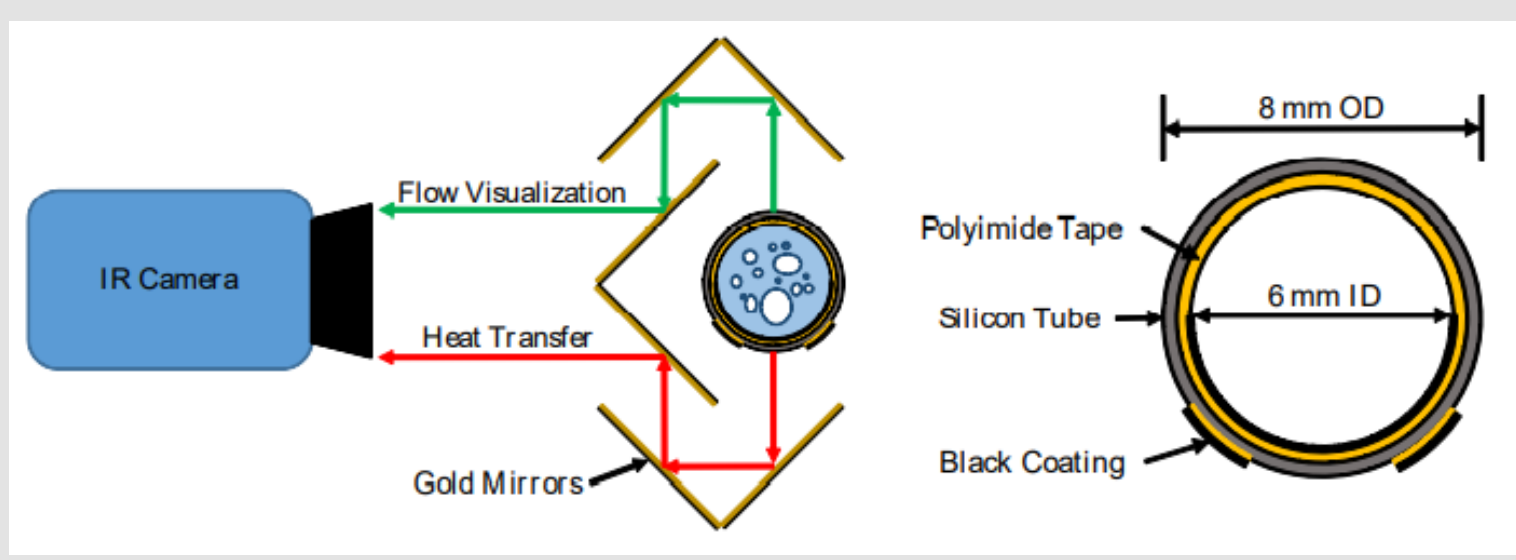

Figure 2: Flow boiling test section setup featuring golden mirrors for simultaneous heat transfer measurements and flow visualization (left image), and cross-sectional view of the flow boiling test section (right image) [21].

Some of the major obstacles associated with IR thermography, pertains to the availability of the IR optical properties, which is required to resolve the temperatures across IR transparent materials, such as silicon and Kapton tape [6,22-24]. Not to mention that IR transparent materials are not easy to machine, such as in the case of $\mathrm{CaF}_{2}$ [25]. In addition, in case there is a sharp temperature gradient, there is a restriction on the minimal number of pixels covering an area, so as to resolve the spatial temperature distribution at a region of interest [22-23]. Jason [23] has also raised an issue pertaining to the integration time with respect to the calibration range, where an error in the order of 10 degrees can incur, in case the measurement was done outside the calibration range.

\section{Time Domain Thermoreflectance}

Another non-contact measurement technique is the time domain thermoreflectance (TDTR), which relies on the examining the material reflectance property variation with temperature. A schematic of the TDTR technique is shown in Figure 3. Such approach is applied in order to determine the thermal properties of materials, which is of interest within the development of new materials, including nanomaterials and thin films. In a study by Mehrvand and Putnam [24], the heat transfer coefficient at the thermal boundary layer within a flowing fluid was examined using TDTR technique. One of the advantages of the TDTR techniques is its spatial resolution relative to IR thermography, due to its use of visible light. On the other hand, TDTR requires a complex setup, which involves various optical equipment and probing instrumentations. Therefore, its generally not applicable to the complex geometries that can arise in boiling heat transfer application.

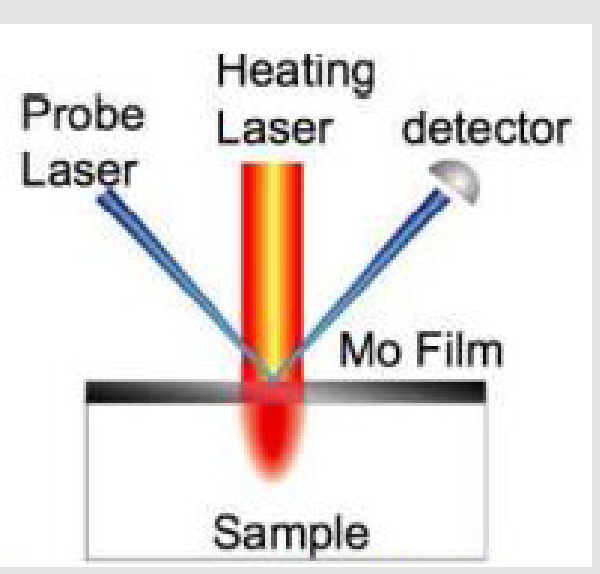

Figure 3: TDTR setup for material thermo conductivity measurement (ww.nist.gov/programs-projects/measurements-and-standards-thermoelectric-materilas). 


\section{Fluorescing Measurement Techniques}

The following subsections summarizes the measurement techniques that utilizes photoluminescent materials, such as liquid crystals and quantum dot. Such measurement approach relies on the spectral emission variation of such materials temperature, which can give a fine spatial resolution of the surface measurement.

\section{Liquid Crystals}

Thermographic techniques based on liquid crystals and fluorophores has been suggested as a temperature indicator. One such an attempt is the work done by Kenning [26], where he examined the wall temperature profile within nucleate boiling application, via a thermochromic liquid crystal. However, such techniques can involve tedious calibration procedures and limitations associated with equipment availability and operating costs [27-30]. Not to mention, the narrow temperature operating range by liquid crystal technique [25].

\section{Quantum Dots}

Quantum dots (QDs) are semiconductors whose length scale is in the order of nanometers and thus subject to 3D confinement. QDs have unique optical properties relative to traditional fluorophores in which it can be excited by a wide range of wavelengths. In addition, it emits light over a narrow spectrum at a longer wavelength, which can easily be captured using a long pass filter. Solid-state lamps in the form of LEDs are usually used for QD excitation purposes. As a direct consequence of the QD length scale, the color of the emitted light can be tuned by changing the QD size via temperature and synthesis time control of the fabrication process. Examples of QDs spectrum emission variation is shown in Figure 4. QDs are prepared in a colloidal liquid, and it can easily be transferred to surfaces of interest via spray or spin coating. Such versatile fabrication processes can greatly downsize the expenses, pertaining to facility complexity [31-38]. Other QDs delivery methods include electrostatic coating, UV curable solutions, as well as sol-gel approach [38-42]. According to the airy diffraction theory, the minimum spatial resolution that can be detected by a camera ( $\mathrm{x}$ ) is related to the observed wavelength $(\lambda)$ by Equation 1. In Equation 1 , $\mathrm{f}$ is the distance between the lens and object and $\mathrm{d}$ is diameter of the aperture. Thus, QD has a spatial resolution advantage over IR thermography, where local heat flux measurements can be acquired at submicron ranges, due to its smaller wavelength emission [43].

$$
x f=1.22 \lambda d(1)
$$

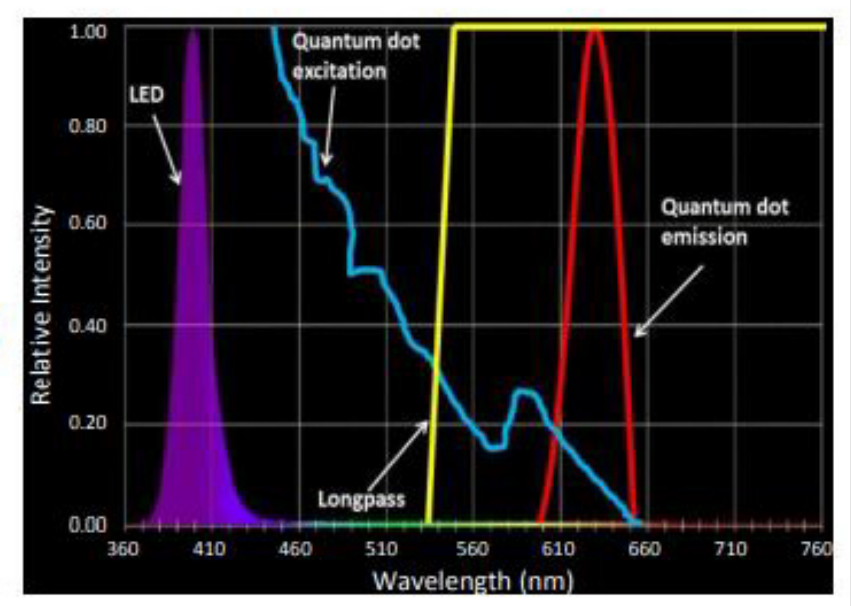

Figure 4: QD, (left) emission from dots of increasing size from left to right (Wikipedia.org/wiki/quantum_dot).(right)Spectral characterization of QD.

Another characteristic of QDs is the temperature dependency of their optical properties as shown in Figure 5, which can be exploited for temperature measurement purposes. This can be related to stoke shift behavior of QDs, which is related to the photoluminescence peak shift, as was observed in previous studies $[33,37,44-46]$. The temperature variation alters the QD optical properties due to thermal expansion of the QD structural lattice
$[30,36]$. Two trends are evident. The intensity of the emitted light tends to decrease with temperature and the peak in the emitted spectrum tends to shift to longer wavelengths. The changes in the intensity and peak wavelength spectra are not necessarily proportional with temperature. The emitted light intensity changes with temperature tend to be quadratic, which makes it less precise than spectral measurement over large temperature ranges $[37,45]$. 
(a)

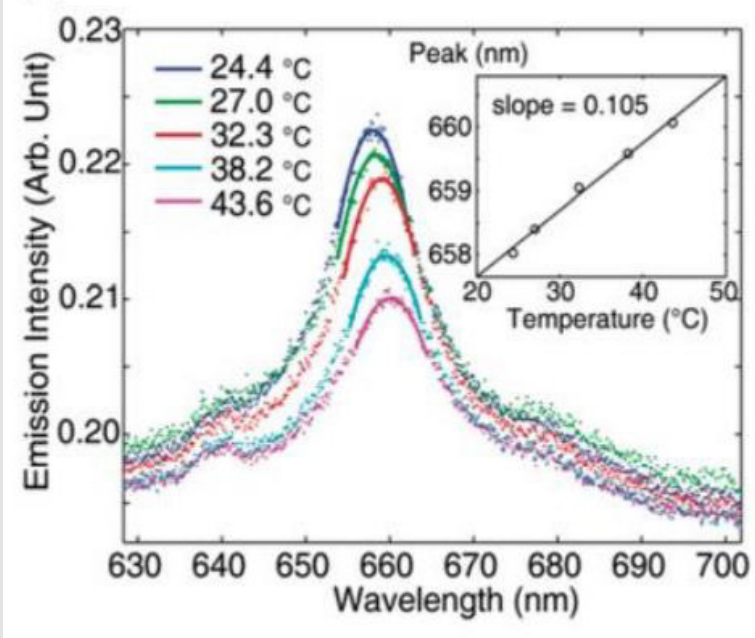

(b)

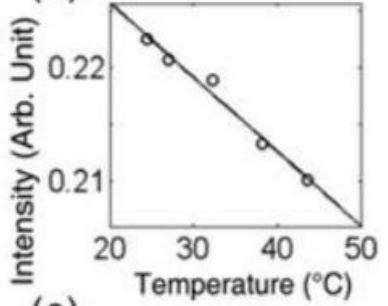

(c)

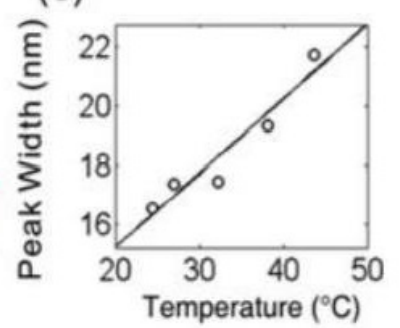

Figure 5: Quantum dot optical property variation with temperature [44].

Relative Intensity

1.00

0.80

0.60

0.40

0.20

0.00

360410460510560610660710760

Wavelength $(\mathrm{nm})$

Several studies have used QDs to measure temperature. Al Hashimi and Kim [47] investigated the local heat flux distribution arising from the vaporization of an ethanol drop, where QDs dispersed within a gelatin film were used to acquire surface temperature variation underlying the ethanol drop. The experimental setup for the ethanol drops temperature measurement is given in Figure 6. Matsuda, et al. [31] used ZnS$\mathrm{AgInS}_{2}$ QDs for surface temperature measurement due to their low toxicity and high temperature sensitivity. Jorge, et al. [33] achieved independency from the excitation source intensity by using multiple QDs with different emission spectra and looking at the intensity ratio. Sakaue, et al. [34] developed a QD temperature sensor for cryogenic application. $\mathrm{Li}$, et al. [44] examined the temperature profile of a micro-heater by calibrating the spectral shift of QDs with temperature. They emphasized the importance of QDs particle concentration on a surface so as to achieve a certain temperature precision as a result of particle size variation [44].
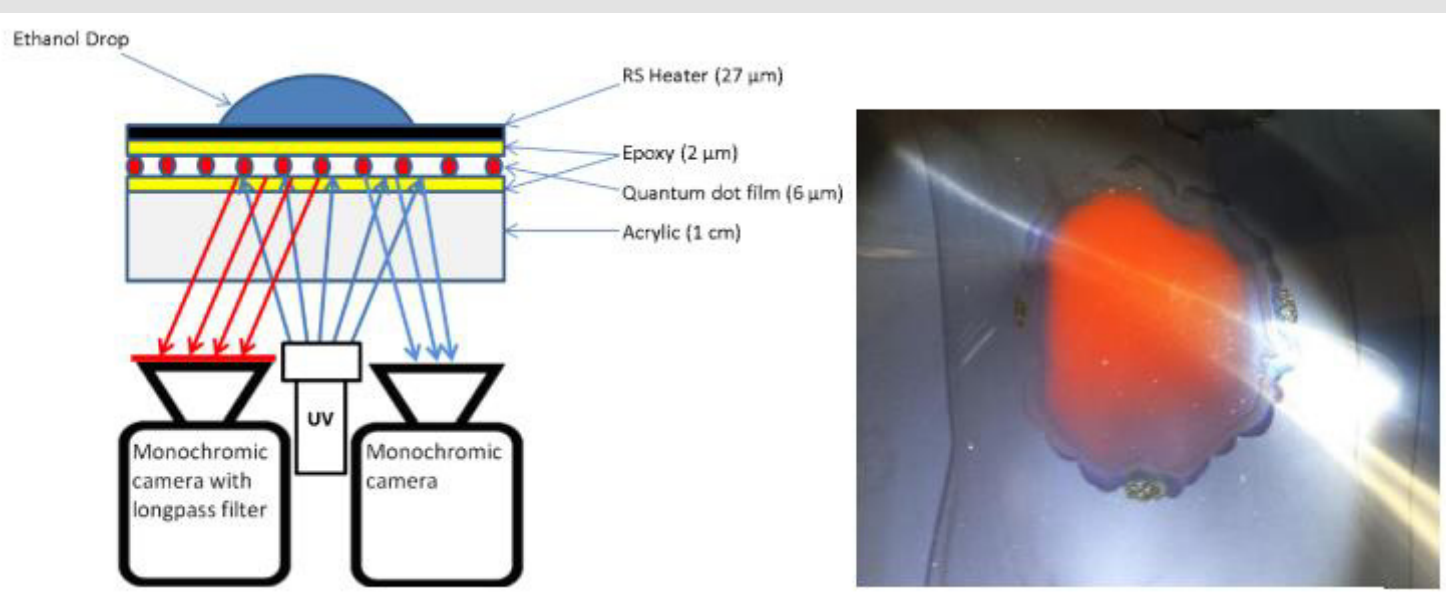

Figure 6: Ethanol drop experimental, (left) test setup, (right)QD-gelatin film. 
The average temperature precision was enhanced at a higher number of QD particles, according to Li et al. [44]. Wang et al. [39] developed a miniaturized temperature sensor in the form of a reflective fiber for high temperature applications. In a similar line of work to Wang et al. [39], Bueno et al. [37] developed a photonic planar waveguide temperature sensor using nanocomposites of CdTe and CdSe, which were embedded in PMMA. QDs-PMMA nanocomposites have a peak spectral emission at a shorter wavelength than colloidal solutions of QDs, due to the particles agglomeration and waveguide effects introduced by PMMA matrix. In addition, PMMA tends to be hydrophobic, which minimizes the effect of humidity upon temperature measurement [37]. One of the issues pertaining to QDs can be related to the photobleaching degradation of the emission, which is associated with the breakdown of QDs as a result of continuous light excitation. Other issues pertain to the temperature calibration, which is sensitive to the observed noise in the QD emission readings. Such issues can be remediated by utilizing a high quantum yield of QDs, or photoluminescence emission, and by utilizing CCD cameras instead of CMOS cameras for higher signal to noise ratio [33-34,48-49] Yu, et al. [45] reported an initial blue shift of the QDs peak spectral emission to shorter wavelengths during the first heating and cooling cycles. Such behavior was resolved by exposing the QDs to several thermal cycles, which resulted in a reproducible spectral peak emission with respect to temperature. Other issues pertain to photooxidation, which can cause an irreversible blue shift due to interaction with surrounding gases [48]. Also, the concentration variation of QDs on a particular area can bring about an uneven intensity distribution, since it's difficult to control the uniformity of QDs over a surface $[38,44]$.

\section{Temperature Sensitive Paint}

Another fluorescing material that works under the same principle as the quantum dots is the temperature sensitive paint (TSP), which comprises of a light emitting luminophores and a binder. As shown in Figure 7, the luminophores gets excited to a higher energy state upon absorption of photons from a short wavelength light source. Afterwards, the excited luminophores undergoes a decay to a lower energy state, where it emits light at a longer wavelength. This process is called photoluminescence. Two conversion processes compete with the photoluminescence of the TSP, which causes the luminophores to decay closer to its ground state: first, the external conversion of the luminophores energy, which is associated with its emitted light quenching via molecular interaction. Such process is relevant within pressure sensitive paint applications, where various oxygen concentrations are associated with different light emission intensity from the luminophores. The second type of the luminophores conversion processes is the internal conversion, which is associated with the energy state variation of luminophore with temperature. Such process is called thermal quenching [25]. The material selection for the luminophores and the binder governs the optical characteristics of the yielded TSP. For instance, the temperature dependency of the luminophore's light emission varies from one material to another. In addition, the operation of the paint as a temperature sensitive or pressure sensitive paint depends on the binder permeability to oxygen [50]. Recent interest in TSP has emerged in several applications, as a local temperature measurement mean.

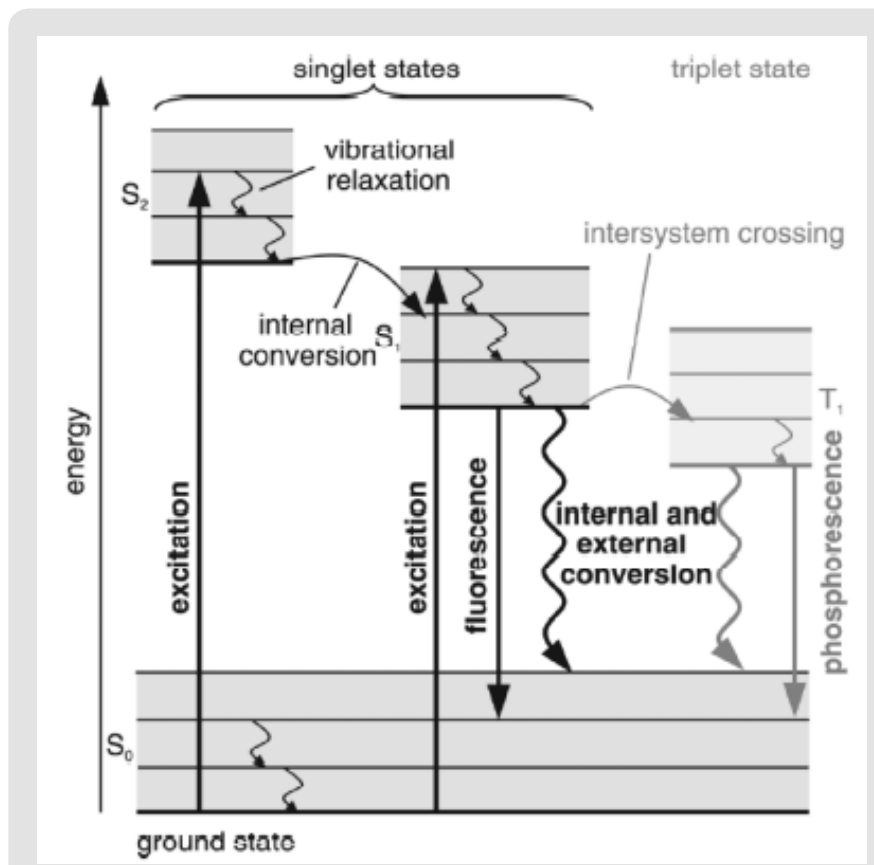

Figure 7: Jablonsky energy-level diagram [25].

One of those attempts is the work by Al Hashimi, et al. [51], where Ruthenium based TSP was used to measure the local heat flux distribution within both pool boiling and flow boiling application. Figures 8 \& 9 illustrate the test section set up within the pool boiling experiment, where the local heat flux distribution was acquired using the inverse heat conduction problem. The temperature measurement across the adhesive layer was used as boundary conductions for the heat conduction problem, where the heat evolved to the boiling fluid was evaluated as the difference between the heat generated at the $\mathrm{NiCr}$ heater and the heat lost to the Sapphire substrate. In Shibuya, et al. [52] study, local temperature variation was captured in form of TSP intensity changes, as a consequence of a passing bubble. Several studies utilized TSP optical properties for surface temperature measurement within wind tunnel facilities some of which were involved in hypersonic flow conditions. 


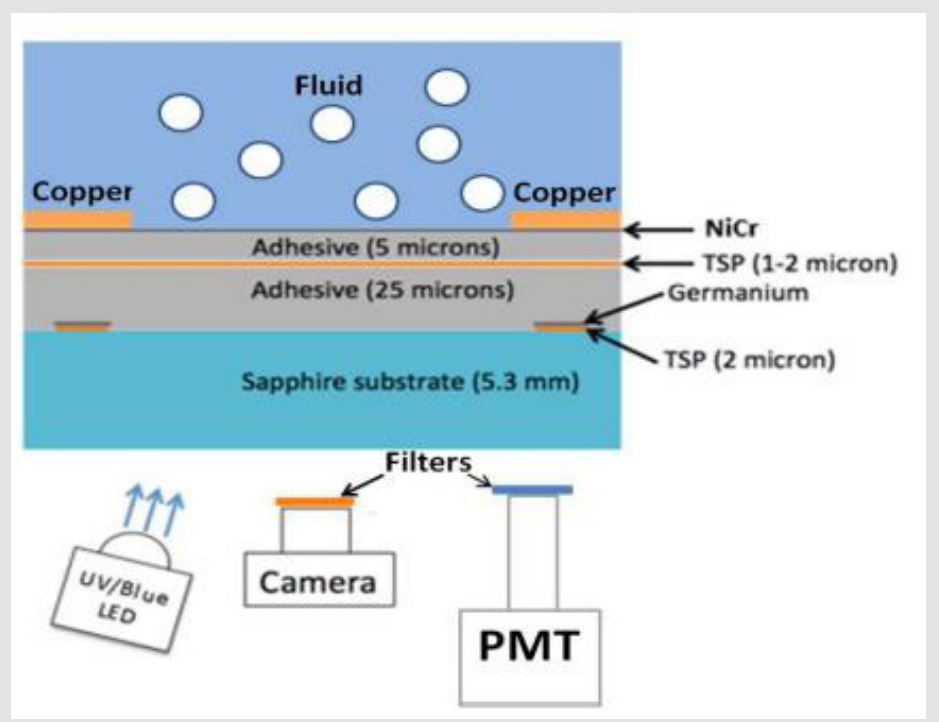

Figure 8: Schematic of the test section.
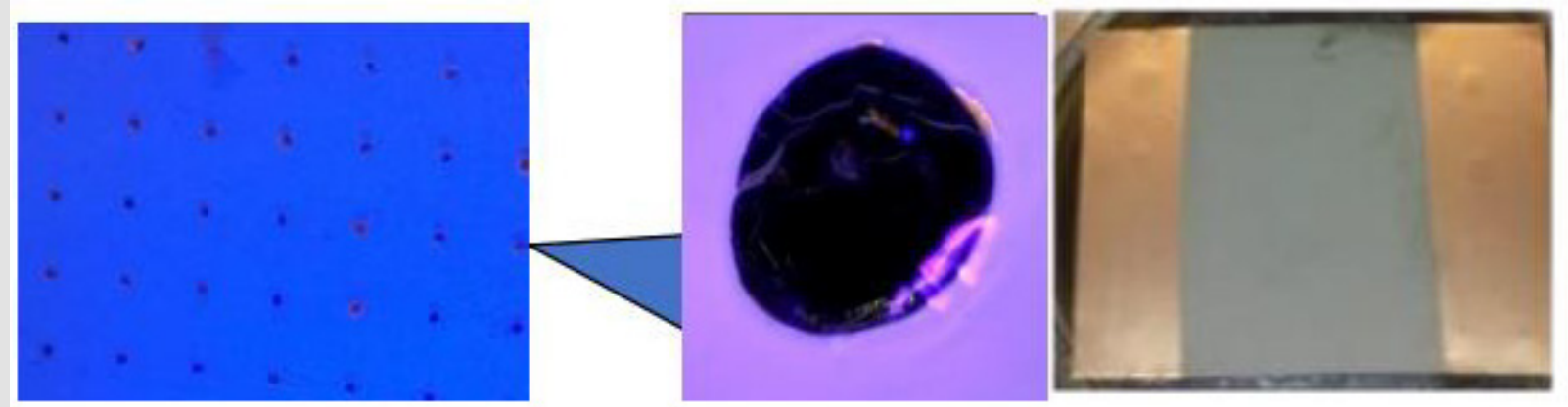

Figure 9: Depiction of the germanium dot and $\mathrm{NiCr}$ heater.

Among the various wind tunnel experiments are the work by Kurits [53], Bhandari [54], Lee, et al. [50], Schramm, et al. [55], Huang [56], Yang, et al. [57] and Ozawa et al. [58], where the local temperature distributions were deduced from surface of interest, based on the observed TSP emission. In more recent study by Liu and Risius [59], TSP is used for thermal imaging purposes within high-enthalpy shock tunnel application. Within Liu and Risius study, a reliable heat flux sensor was used in conjunction with an in-situ calibration technique, so as to determine the thermal penetration within the TSP layer, via an analytical inverse solution [59]. Long [60] utilized multiple luminophores one of which is temperature insensitive, so as to account for the intensity variation within the excitation light source. In a study by Huang, et al. [61] examined the temperature profile within a microchannel flow application, via the optical properties of the TSP. Simultaneous flow visualization and thermal profiling was achieved by Matsuda, et al. [62] for a multiphase flow phenomenon, within microchannel applications. In Matsuda study, TSP was used to survey the temperature profile at various flow boiling conditions, where the acquired Nusselt number was in good agreement with the SiederTate equation [62]. Other work by Ishii and Fumoto [63] utilized TSP in order to acquire temperature distribution at the evaporator wall of a pulsating heat pipe. From the temperature data analysis, a correlation was acquired between the temperature distribution and the observed oscillatory flow phenomenon within the pipe. In addition, a temperature accuracy of $0.263^{\circ} \mathrm{C}$ was reported within Ishii and Fumoto study [63].

Recent interest in luminophores utilization was found in other applications, such as its usage as PSP. Examples are the studies carried out by Jiao, et al. [64,66,67]. In Jiao, et al. [64] study, a twodimensional correction factor was adopted in the data analysis, so as to resolve the temperature sensitivity of the PSP. Such result was validated against CFD data. In another study by Noda, et al. [66], PSP was used to resolve the transient pressure field on a NACA 0012 airfoil. Other applications for PSP include jet impingement, such as the study carried out by Li, et al. [67], where it was used 
to examine the pressure field characteristics near the impingement point, such as the nozzle-plate distance, impingement angle, and pressure ratio. The luminophores suffer from similar shortcomings as the quantum dots. For instance, paint thickness inhomogeneities over the surface as well as non-uniform excitation light illumination are among several factors attributed to the observed noise within optical measurements. Background noise and particulates can also interfere with the optical properties of the TSP, which can bring about a shift in the observed intensities. Photobleaching effect tends to be more severe in the case of TSP [68]. In a recent study by Liu et al [69], the temperature dependency of the thermal diffusivity parameter was addressed within the TSP heat flux measurement, which can arise within hypersonic wind tunnel applications. A correction factor was developed within Liu et al study, which was validated against simulation data [69]. Other issues pertaining to the error arising from the TSP apparent temperature relative to the actual wall temperature, as was suggested in a study by Liu, et al. [70]. Such discrepancy becomes more prominent as the thickness of the TSP layer increases, relative to adjacent layers [70].

\section{Conclusion}

In the current review paper, various temperature measurement techniques were examined, along with their applications in various research works. Conventional temperature measurement techniques include thermocouple and microheater arrays, where thermocouples can misrepresent the local interaction at the measurement surface, due to its spatial limitation by its average measurement approach. On the other hand, microheaters are complex to fabricate and are difficult to install on non-flat geometries, such as tubes. In the past decade, IR thermography was adaptedas a measurement technique, which can monitor the temperature distribution at a micron resolution. Therefore, an enhanced measurement fidelity can be achieved with respect to the investigated phenomenon. Some of the drawbacks of the IR technique is its compatibility with the measurement surface, which can be opaque to the IR wavelength or are generally expensive to acquire. Novel temperature measurement approaches involve fluorescing materials, which act as a potential alternative to IR thermography. Such materials include QDs and TSP, which operate within the visible wavelength. Hence, a broader range of materials can be used as a substrate for the experiment, where an affordable monochromic camera can be used for local temperature measurement purposes in various applications, such as pool boiling.

\section{Conflict of Interest}

No conflict of interest with any institution/organization.

\section{References}

1. Sobac, A, Brutin D (2010) Heat Transfer and Flow Instabilities in Ethanol Sessile Drops Under Evaporation. Proceedings of the $14^{\text {th }}$ International Heat Transfer Conference, Washington, DC, USA, ASME Paper No. IHTC14-22185.

2. Chu H, Yu B (2009) A new comprehensive model for nucleate pool boiling heat transfer of pure liquid at low to high heat fluxes including CHF. International Journal of Heat and Mass Transfer 52: 4203-4210.

3. Narumanchi S, Troshko A, Bharathan D, Hassani V (2008) Numerical simulations of nucleate boiling in impinging jets: Applications in power electronics cooling. International Journal of Heat and Mass Transfer 51: $1-12$.

4. Pezo M, Stevanovic V (2011) Numerical prediction of critical heat flux in pool boiling with the two-fluid model. International Journal of Heat and Mass Transfer 54: 3296-3303.

5. Theofanous TG, Tu JP, Dinh AT, Dinh TN (2002) The boiling crisis phenomenon Part I: nucleation and nucleate boiling heat transfer. Experimental Thermal and Fluid Science 26: 775-792.

6. Kim TH, Kommer E, Dessiatoun S, Kim J (2012) Measurement of twophase flow and heat transfer parameters using infrared thermometry. International Journal of Multiphase Flow 40: 56-67.

7. Truong E (2013) Cryogenic Single-Phase Heat Transfer in a Microscale Pin Fin Heat Sink. BS. Thesis, OSU, USA.

8. Chen Q, Luo X, Zhou S, Liu S (2011) Dynamic junction temperature measurement for high power light emitting diodes.Review of scientific instruments 82 .

9. Kaltsas G, Petropoulos A, Tsougeni K, Pagonis DN, Speliotis T, et al. (2007) A novel microfabrication technology on organic substrates -Application to a thermal flow sensor. Journal of Physics: Conference Series 92.

10. Abad E, Mazzolai B, Juarros A, Mondini A, Krenkow A, et al. (2010) Fabrication and Encapsulation Processes. Radio Frequency Identification Fundamentals and Applications, Design Methods and Solutions, pp. 237251.

11. Lee G, Huang F, Lee C, Miau J (2004) A New Fabrication Process for a Flexible Skin with Temperature Sensor Array and Its Application. ACTA Mechanica Sinica 20(2)

12. Lee G, Wu J, Miau J (2002) A New Fabrication Process for a Flexible Skin with Temperature Sensor Array. Journal of the Chinese Institute of Engineers 25(6): 619-625.

13. Yusoff A, Syahrul M, Henkel K (2007) Film adhesion in amorphous silicon solar cells. Bull Mater Sci 30(4): 329-331.

14. (2011) Nickel as a Coating Material, 2011, Materion Brush Performance Alloys: Technical Tidbits (34).

15. Guereca GR (2007) Explosive vaporization in microenclosures and boiling phenomena on submicron thin film strip heaters. DScTech, Universidad Carlos III de Madrid, Spain.

16. Demiray F, Kim J (2004) Microscale heat transfer measurement during pool boiling of FC-72: effect of subcooling. International Journal of Heat and Mass Transfer 47: 3257-3268.

17. Xiao S, Che L, Li X, Wang Y (2007) A cost-effective flexible MEMS technique for temperature sensing. Microelectronics Journal 38: 360364.

18. Chia BT, Chang D, Liao H, Yang Y, Shih W, et al. (2007) Temperature Sensor Array Using Flexible Substrate. MEMS 589-592. 
19. Petropoulos A, Goustouridis D, Speliotes T, Kaltsas G (2009) Demonstration of a New Technology Which Allows Direct Sensor Integration on Flexible Substrates. The European Physical Journal Applied Physics 46(1).

20. Siegel J, Lyutakov O, Rybka V, Kolska Z, Svorcik V (2011) Properties of gold nanostructures sputtered on glass. Nanoscale Research Letters $6(96)$.

21. Scammell A, Kim J (2015) Heat transfer and flow characteristics of rising Taylor bubbles. International Journal of Heat and Mass Transfer 89: 379389.

22. Solotych V, Kim J, Dessiatoun SV (2014) Local Heat Transfer Measurements within a Representative Plate Heat Exchanger Geometry Using Infrared (IR) Thermography. Journal of Enhanced Heat Transfer 21(4-5): 353-372.

23. Thompson JC (2015) A Study on Critical Heat Flux Mechanisms and the Transition to Film Boiling. MS. Thesis, UMD, College Park, USA.

24. Mehrvand M, Putnam SA (2017) Probing the Local Heat Transfer Coefficient of Water-Cooled Microchannels Using Time-Domain Thermoreflectance. Journal of Heat Transfer 139.

25. Lorenz M, Horbach T, Schulz A, Bauer J (2013) A Novel Measuring Technique Utilizing Temperature Sensitive Paint-Measurement Procedure, Validation, Application, and Comparison with Infrared Thermography. Journal of Turbomachinery 135 .

26. Kenning DBR (1992) Wall temperature patterns in nucleate boiling. International Journal of Heat Mass Transfer 35(1): 73-86.

27. Farina DJ (1995) Making surface temperature measurements using liquid crystal thermography. Test \& Measurement, Electronic-Cooling.

28. Azar K, Benson JR (1991) Liquid Crystal Imaging for Temperature Measurement of Electronic Devices. Seventh IEEE SEMI-THERMTM Symposium, p. 23-33.

29. Lou J, Hatton TA, Laibinis PE (1997) Fluorescent Probes for Monitoring Temperature in Organic Solvents. Analytical Chemistry 69(6): 12621264.

30. Angell JJ (2011) Synthesis and Characterization of Cdse-Zns Coreshell Quantum Dots. MS. Thesis, California Polytechnic State University, USA.

31. Matsuda Y, Torimoto T, Kameya T, Kameyama T, Kuwabata S, et al (2013) ZnS-AgInS2 nanoparticles as a temperature sensor," Sensors and Actuators B: Chemical 176: 505-508.

32. Gui C, Wang K, Li C, Dai X, Cui D (2014) A CCD-based reader combined with CdS quantum dot-labeled lateral flow strips for ultrasensitive quantitative detection of CagA. Nanoscale Research Letters, Springer open Journal.

33. Jorge PAS, Mayeh M, Benrashid R, Caldas P, Santos J L, et al. (2006) Quantum dots as self-referenced optical fibre temperature probes for luminescent chemical sensors," Measurement Science and Technology 17: $1032-1038$

34. Sakaue H, Aikawa A, Iijima Y, Kuriki T, Miyazaki T (2012) Quantum Dots as Global Temperature Measurements.Quantum Dots-A variety of New Applications, pp. 137-152,

35. Murray C, Kagan C, Bawendi M (2000) Synthesis and Characterization of Monodisperse Nanocrystals and Close-Packed Nanocrystal Assemblies. Annual Review Material Science 30: 545-610.

36. Baskoutas S, Terzis A (2006) Size-dependent band gap of colloidal quantum dots. Journal of Applied Physics 99.

37. Bueno A, Suarez I, Abargues R, Sales S, Pastor JPM (2012) Temperature Sensor Based on Colloidal Quantum Dots-PMMA Nanocomposite Waveguides. In: E. H. Yang (Eds.)., IEEE Sensors Journal 12(10): 30693074
38. Liu W, Zhang Y, Wu H, Feng Y, Zhang T, et al. (2014) Planar temperature sensing using heavymetal-free quantum dots with micrometer resolution. Nanotechnology, 25, IOP Publishing Ltd, UK.

39. Wang H, Yang A, Chen Z, Geng Y (2014) Reflective Photoluminescence Fiber Temperature Probe Based on the CdSe/ZnS Quantum Dot Thin Film. Optics and Spectroscopy 117(2): 235-239.

40. Bastida Gd, Arregui FJ, Goicoechea J, Matias IR (2006) Quantum DotsBased Optical Fiber Temperature Sensors Fabricated by Layer-byLayer. IEEE Sensors Journal 6(6): 1378-1379.

41. He-lin W, Ai-jun Y, Cheng-hua S (2013) Luminescent high temperature sensor based on the CdSe/ZnS quantum dot thin film. Optoelectronics Letters 9(6): 421-424.

42. Larrion B, Hernaez M, Arregui FJ, Goicoechea J, Bravo J, et al. (2009) Photonic Crystal Fiber Temperature Sensor Based on Quantum Dot Nanocoatings. Journal of Sensors, Hindawi Publishing Corporation.

43. Shepherd M (2012) Correct Sampling of Diffraction Limited Images. California Institute of Technology, online document.

44. Li S, Zhang K, Yang J, Lin L, Yang H (2007) Single Quantum Dots as Local Temperature Markers. Nano Letters 7(10): 3102-3105.

45. Yu HCY, Leon-Saval SG, Argyros A, Barton GW (2010) Temperature effects on emission of quantum dots embedded in polymethylmethacrylate. Applied Optics 49(15).

46. Valerini D, Cretí A, Lomascolo M (2005) Temperature dependence of the photoluminescence properties of colloidal $\mathrm{CdSe} / \mathrm{ZnS}$ core/shell quantum dots embedded in a polystyrene matrix," Physical Review B 71

47. Al Hashimi H, Kim J (2016) Quantum Dot Temperature Sensor Ab Initio Test: Droplet Vaporization Heat Transfer. In ASME 2016 Heat Transfer Summer Conference collocated with the ASME 2016 Fluids Engineering Division Summer Meeting and the ASME 2016 14th International Conference on Nanochannels, Microchannels, and Minichannels (pp. V002T08A012-V002T08A012). American Society of Mechanical Engineers.

48. Shen Y (2008) Photoluminescence spectral study of single CdSe/ZnS ColloidalNanocrystals in Poly (methyl methacrylate) and Quantum Dots molecules. Ph.D. thesis, UC, San Diego, local identifier: b6636099.

49. Maruyama H, Masuda T, Arai F (2011) Local Temperature Measurement and Control Using Functional Gel-Tool Containing a Quantum Dot by Color Analysis Of Fluorescence Spectrum. $15^{\text {th }}$ International Conference on Miniaturized Systems for Chemistry and Life Sciences, pp. 19531955.

50. Lee JJ, Dutton JC, Jacobi AM (2007) Application of TemperatureSensitive Paint for Surface Temperature Measurement in Heat Transfer Enhancement Applications. Journal of Mechanical Science and Technology 21: 1253-1262.

51. Al Hashimi H, Hammer C, Lebon M, Zhang D, Kim J (2017) Phase Change Heat Transfer Measurements Using Temperature Sensitive Paints. Journal of Heat Transfer ASME

52. Shibuya A, Ueki R, Suzuki Y, Tange (2016) Temporal Temperature Distribution Measurement of a Heat Transfer Surface Of A Flow Boiling Heat Sink With A Micro-Gap Using Temperature Sensitive Paint," Proceedings of the First Pacific Rim Thermal Engineering Conference.

53. Kurits I (2008) Quantitative Global Heat-Transfer Measurements Using Temperature Sensitive Paint on A Blunt Body In Hypersonic Flows. MS. Thesis, UMD, College Park, Maryland, USA.

54. Bhandari P (2012) Evaluation and Improvement of Temperature Sensitive Paint Data Reduction Process Through Analysis of Tunnel Data. MS. Thesis, UMD, College Park, USA. 
55. Schramm JM, Hannemann K, Ozawa H, Beck W, Klein C (2015) Development of Temperature Sensitive Paints for the High Enthalpy Shock Tunnel Gottingen, HEG. $8^{\text {th }}$ European Symposium on Aerothermodynamics for Space Vehicles.

56. Huang C (2005) Molecular Sensors for MEMS," PhD. Thesis, Purdue University Graduate School, West Lafayette, Indiana, USA.

57. Yang L, Erdem E, Kontis K (2011) Application of Pressure-and TemperatureSensitive Paint in a Hypersonic Double Ramp Flow. 28 International Symposium on Shock Waves, pp. 759-765.

58. Ozawa H, Laurence SJ, Martinez Schramm J, Wagner A, Hannemann K (2014) Fast-response temperature-sensitive-paint measurements on a hypersonic transition cone. International Journal of Multiphase Flow 56 (2015).

59. Liu T, Risius S (2019) In-situ calibration for temperature-sensitive-paint heat-flux measurement on a finite base. International Journal of Heat and Mass Transfer 140: 420-425

60. Long SR (2011) A Temperature-Corrected Dual-Luminophore PressureSensitive Paint System. BS. Thesis, Ohio State University, USA.

61. Huang C, Li C, Wang H, Liou T (2013) The application of temperaturesensitive paints for surface and fluid temperature measurements in both thermal developing and fully developed regions of a microchannel. Journal of Micromechanics and Microengineering.

62. Matsuda Y, Kawanami O, Orimo R, Uete K, Watanabe A, et al. (2020) Simultaneous measurement of gas-liquid interface motion and temperature distribution on heated surface using temperature-sensitive paint. International Journal of Heat and Mass Transfer 153.

ISSN: 2574-1241

DOI: $10.26717 /$ BJSTR.2021.40.006465

Omar Chaalal. Biomed J Sci \& Tech Res

cC) (P) This work is licensed under Creative Commons Attribution 4.0 License

Submission Link: https://biomedres.us/submit-manuscript.php
63. Ishii K, Fumoto K (2019) Temperature visualization and investigation inside evaporator of pulsating heat pipe using temperature-sensitive paint. Applied Thermal Engineering 155: 575-583.

64. Jiao L, Liu X, Shi Z, Zhang W, Liang L, et al. (2020) A two-dimensional temperature correction method for pressure-sensitive paint measurement on helicopter rotor blades. Experiments in Fluids 61(104).

65. Peng D, Chen J, Jiao L, Liu Y (2018) A fast-responding semi-transparent pressure-sensitive paint based on through-hole anodized aluminum oxide membrane. Sensors and Actuators A: Physical 274.

66. Noda T, Nakakita K, Wakahara M, Kameda M (2018) Detection of smallamplitude periodic surface pressure fluctuation by pressuresensitive paint measurements using frequency-domain methods. Experiments in Fluids 59 (94).

67. Li X, Liu X, Liu Y, Peng D (2020) Experimental study of near-wall underexpanded jet impingement on a flat plate using temperatureinsensitive semi-transparent pressure-sensitive paint. Experiments in Fluids 61(232).

68. Kose ME (2005) Multi-Luminophore Coatings For Pressure Sensitive Paint Applications. PhD. Thesis, University of Florida, USA.

69. Liu T, Montefort J, Schick N, Stanfield S, Palluconi S, et al. (2019) Correction for effect of temperature-dependent diffusivity on temperature-sensitive-paint heatflux measurement. International Journal of Heat and Mass Transfer 137: 337-348.

70. Liu X, Shao H, Zhou W, Liu Y, Peng D (2020) Apparent temperature in temperature-sensitive paint measurement and its effect on surface heat flux determination for hypersonic flows. Measurement Science and Technology 31(12).

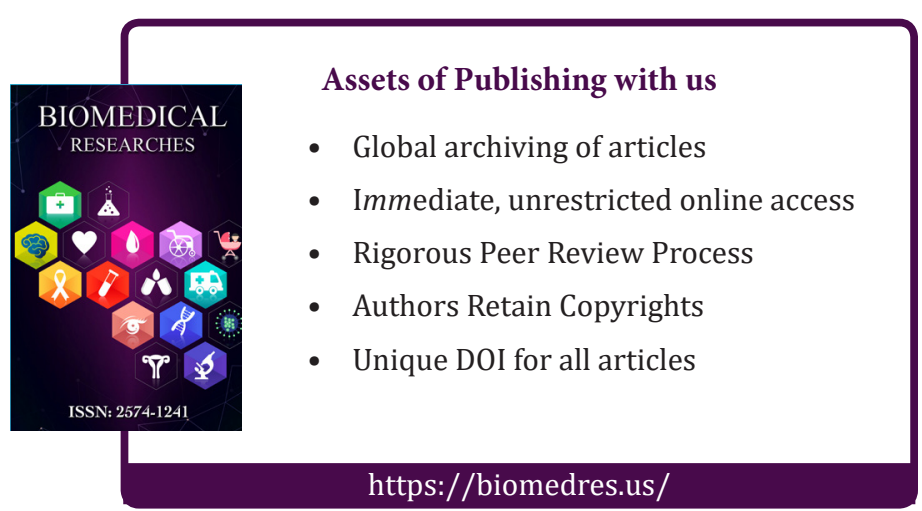

